\title{
Benefits of physical activity on COPD hospitalisation depend on intensity
}

\author{
David Donaire-Gonzalez ${ }^{1,2,3,4}$, Elena Gimeno-Santos ${ }^{1,2,3}$, Eva Balcells ${ }^{3,5,6,7}$, \\ Jordi de Batlle ${ }^{8}$, Maria A. Ramon ${ }^{5,9,10}$, Esther Rodriguez ${ }^{5,9,10}$, Eva Farrero ${ }^{11,12}$ \\ Marta Benet ${ }^{1,2,3}$, Stefano Guerra 1,2,3,13 , Jaume Sauleda ${ }^{5,14,15}$, Antoni Ferrer 3,5,7, \\ Jaume Ferrer ${ }^{5,9,10}$, Joan A. Barberà ${ }^{5,16}$, Robert Rodriguez-Roisin ${ }^{5,16}$, \\ Joaquim Gea $3,5,6,7$, Alvar Agustí ${ }^{5,16}$, Josep M. Antó1,2,3,6, \\ Judith Garcia-Aymerich ${ }^{1,2,3}$ and the PAC-COPD Study Group ${ }^{17}$
}

Affiliations: ${ }^{1}$ Centre for Research in Environmental Epidemiology (CREAL), Barcelona, Spain. ${ }^{2}$ CIBER Epidemiología y Salud Pública (CIBERESP), Barcelona, Spain. ${ }^{3}$ Departament de Ciències Experimentals i de la Salut, Universitat Pompeu Fabra, Barcelona, Spain. ${ }^{4}$ Physical Activity and Sports Sciences Department, Fundació Blanquerna, Barcelona, Spain. ${ }^{5}$ CIBER de Enfermedades Respiratorias (CIBERES), Madrid, Spain. ${ }^{6}$ Hospital del Mar Medical Research Institute (IMIM), Barcelona, Spain. ${ }^{7}$ Department of Pneumology, Hospital del Mar IMIM, Barcelona, Spain. ${ }^{8}$ Section of Nutrition and Metabolism, International Agency for Research on Cancer (IARC), Lyon, France. ${ }^{9}$ Department of Pneumology, Hospital Universitari Vall d'Hebron, Barcelona, Spain. ${ }^{10}$ Departament de Medicina, Universitat Autònoma de Barcelona, Barcelona, Spain. ${ }^{11}$ Department of Pneumology, Hospital Universitari de Bellvitge, Barcelona, Spain. ${ }^{12}$ Institut d'Investigació Biomèdica de Bellvitge (IDIBELL), Barcelona, Spain. ${ }^{13}$ Arizona Respiratory Center, University of Arizona, Tucson, AZ, USA. ${ }^{14}$ Department of Pneumology, Hospital Universitari Son Espases, Palma de Mallorca, Spain. ${ }^{15}$ Institut d'Investigació Sanitària de Palma (IdISPa), Palma de Mallorca, Spain. ${ }^{16}$ Servei de Pneumologia (Thorax Institute), Hospital Clínic, Institut d'Investigacions Biomèdiques August Pi i Sunyer (IDIBAPS), Universitat de Barcelona, Barcelona, Spain. ${ }^{17} \mathrm{~A}$ full list of the PAC-COPD Study Group members and their affiliations can be found in the Acknowledgements section.

Correspondence: Judith Garcia-Aymerich, Centre for Research in Environmental Epidemiology (CREAL), Dr. Aiguader 88, 08003 Barcelona, Spain. E-mail: jgarciadcreal.cat

ABSTRACT The present study aims to disentangle the independent effects of the quantity and the intensity of physical activity on the risk reduction of chronic obstructive pulmonary disease (COPD) hospitalisations.

177 patients from the Phenotype Characterization and Course of COPD (PAC-COPD) cohort (mean $\pm \mathrm{SD}$ age $71 \pm 8$ years, forced expiratory volume in $1 \mathrm{~s} 52 \pm 16 \%$ predicted) wore the SenseWear Pro 2 Armband accelerometer (BodyMedia, Pittsburgh, PA, USA) for eight consecutive days, providing data on quantity (steps per day, physically active days and daily active time) and intensity (average metabolic equivalent tasks) of physical activity. Information on COPD hospitalisations during follow-up ( $2.5 \pm 0.8$ years) was obtained from validated centralised datasets.

During follow-up 67 (38\%) patients were hospitalised. There was an interaction between quantity and intensity of physical activity in their effects on COPD hospitalisation risk. After adjusting for potential confounders in the Cox regression model, the risk of COPD hospitalisation was reduced by $20 \%$ (hazard ratio (HR) $0.79,95 \%$ CI $0.67-0.93 ; p=0.005$ ) for every additional 1000 daily steps at low average intensity. A greater quantity of daily steps at high average intensity did not influence the risk of COPD hospitalisations (HR 1.01, p=0.919). Similar results were found for the other measures of quantity of physical activity.

Greater quantity of low-intensity physical activity reduces the risk of COPD hospitalisation, but highintensity physical activity does not produce any risk reduction.

@ERSpublications

Greater quantity of low-intensity physical activity reduces the risk of COPD hospitalisation http://ow.ly/Oe2RE

This article has supplementary material available from erj.ersjournals.com

Received: Sept 172014 | Accepted after revision: May 282015 | First published online: July 232015

Copyright OERS 2015 


\section{Introduction}

Reducing the frequency of exacerbations in patients with chronic obstructive pulmonary disease (COPD) is a major therapeutic goal [1], since exacerbations accelerate decline in lung function and exercise capacity and jeopardise both quality of life and survival in these patients [1-3]. Moreover, COPD exacerbations are the most frequent cause of medical consultation and hospitalisation in COPD and cause $50-75 \%$ of the healthcare costs associated with COPD.

Currently, tobacco smoking cessation and pharmacological treatment of COPD reduce and prevent COPD exacerbations, but lifestyle factors, such as physical activity or diet also modulate them beneficially [3-5]. The evidence from prospective studies supporting the beneficial effects of physical activity in preventing COPD exacerbations is consistent across studies involving different physical activity measures, severity of disease and geographical areas [6]. Yet common limitations of all these previous studies are the lack of control for the previous history of COPD exacerbations, which is now known to be the best predictor of further exacerbations [7], and, in most cases, the lack of an objective physical activity assessment. Similarly, whether these beneficial effects of physical activity are due to its quantity and/or its intensity has never been assessed, despite the fact that intensity of physical activity has been proven important for health benefits in other diseases $[8,9]$. This is important because it may give guidance to the implementation of research findings in clinical practice, since no COPD-specific recommendations for an adequate physical activity are available so far.

We hypothesised that both higher quantity and intensity of physical activity could prevent COPD hospitalisations. To test this hypothesis, we assessed the independent effects of the quantity and intensity of objectively measured physical activity on the risk of hospitalisation due to a COPD exacerbation in the Phenotype Characterization and Course of COPD (PAC-COPD) study cohort [10].

\section{Methods}

Study design, participants and ethics

This is a prospective longitudinal study nested in the PAC-COPD project, whose methodology and main results have been reported previously [10]. In brief, COPD patients were enrolled during their first hospitalisation due to COPD exacerbation in nine teaching hospitals in Spain. Patients were evaluated 3 months after discharge, when clinically stable (baseline). The diagnosis of COPD was established according to the American Thoracic Society/European Respiratory Society (ERS) guidelines. Patients were invited to participate in a second visit for follow-up assessment 18-24 months later, while clinically stable. In this second visit, a sample of 177 patients, representative of the PAC-COPD cohort [11], accepted the monitoring of their physical activity with an accelerometer (see later). All patients were followed-up until December 31, 2010 or date of death, whichever came first (median 2.6 years). The ethics committees of all the participating hospitals approved the study, and written informed consent was obtained from each participant.

\section{Measurements}

Patients were instructed to wear an accelerometer (SenseWear Pro 2 Armband; BodyMedia, Pittsburgh, PA, USA) placed on the right arm during waking hours (08:00-10:00 h) over eight consecutive days. On average, they wore the accelerometer for 6 days and recorded a mean $95 \%$ of daytime hours $(13.5 \mathrm{~h}$ of a maximum $14 \mathrm{~h}$ ) [11]. The quantity of physical activity was assessed using 1) the number of steps per day; 2) the number of days per week that the patient could be considered physically active, operationally defined by $\geqslant 5000$ steps per day [12]; and 3) time (minutes) per day spent in physical activity (defined as any minute with $\geqslant 1.5$ metabolic equivalent tasks (METs)). The intensity of physical activity was assessed by the average METs over the time spent in physical activity.

Support statement: Supported by Fondo de Investigación Sanitaria (FIS PI052292) and the Spanish Society of Pneumology and Thoracic Surgery (SEPAR 2004/136). The Phenotype Characterization and Course of COPD (PAC-COPD) study is funded by grants from Fondo de Investigación Sanitaria (FIS PI020541), Ministry of Health, Spain; Agència d'Avaluació de Tecnologia i Recerca Mèdiques (AATRM 035/20/02), Catalonia Government; the Spanish Society of Pneumology and Thoracic Surgery (SEPAR 2002/137); the Catalan Foundation of Pneumology (FUCAP 2003 Beca Marià Ravà); Red RESPIRA (RTIC C03/11); Red RCESP (RTIC C03/09); Fondo de Investigación Sanitaria (PI052486); Fondo de Investigación Sanitaria (PI052302); Fundació La Marató de TV3 (041110); DURSI (2005SGR00392); and unrestricted educational grants from Novartis Farmacèutica (Spain) and AstraZeneca Farmacéutica (Spain). CIBERESP (CIBER Epidemiología y Salud Pública) and CIBERES (CIBER de Enfermedades Respiratorias) are funded by the Instituto de Salud Carlos III, Ministry of Health, Spain. There is no involvement of funding sources in study design; in the collection, analysis, and interpretation of data; in the writing of the report; nor in the decision to submit the article for publication. Researchers are independent from funders. Funding information for this article has been deposited with FundRef.

Conflict of interest: Disclosures can be found alongside the online version of this article at erj.ersjournals.com 
The information about COPD hospitalisations (dates and diagnoses at discharge) during the 12 months prior to enrolment (from 2005 to 2006) and during follow-up (from 2006 until December 31, 2010) was obtained from a national administrative database (online supplementary material).

Other measurements included sociodemographic factors, pharmacological and nonpharmacological treatment, smoking habit, dietary habits, comorbidities, health-related quality of life, dyspnoea, complete lung function (including forced spirometry, body plethysmography, diffusing capacity and arterial blood gases), nutritional status, exercise capacity, lung density and structure and systemic inflammation (online supplementary material).

\section{Statistical analysis}

Provided that sample size was fixed by the primary scientific objectives of the PAC-COPD study, prior to any analysis we calculated the statistical power to answer our research question with 177 patients using the software GRANMO 7.10 (www.imim.cat/ofertadeserveis/en_granmo.html). Accepting an $\alpha$ risk of 0.05 in a two-sided test, and using our own data about the distribution of physically active:physically inactive COPD patients [11], the statistical power to recognise as statistically significant a risk reduction in COPD hospitalisation was $84 \%$, similar to that reported in previous literature [6].

The relationship between baseline physical activity outcomes and risk of COPD hospitalisation was first tested using the Kruskal-Wallis test and then using Cox proportional hazard regression models. To test the independent effect of quantity and intensity of physical activity, we stratified the association between the three outcomes of quantity of physical activity and COPD hospitalisation according to the average of intensity of physical activity (categorised by its median value in our population (2.7 METs)). Because the effect of quantity variables was restricted to the low-intensity group, multivariate models included an interaction term between each variable of physical activity quantity and intensity (see Results). The treatment of potential confounders and the goodness-of-fit tests are detailed in the online supplementary material. Final models were stratified according to 1$)$ severity of airflow limitation $(\geqslant 50 \%$ and $<50 \%$ forced expiratory volume in $\left.1 \mathrm{~s}\left(\mathrm{FEV}_{1}\right)\right)$ [2] and 2) having had a COPD hospitalisation in the previous year. We performed additional analysis using the volume of physical activity $\left(\right.$ METs $\cdot \mathrm{min}^{-1} \cdot$ day $^{-1}$ ) as the exposure. As sensitivity analyses we 1) excluded subjects with extreme values ( $>95$ th percentile) in the accelerometer measures; 2) used COPD hospitalisation or all-cause mortality as the outcome; 3) used all-cause hospitalisation as the outcome; and 4) categorised intensity of physical activity in three groups. All analyses were conducted using R 3.0.1 (R Foundation for Statistical Computing, Vienna, Austria).

\section{Results}

We enrolled 177 COPD patients (94\% male, mean \pm SD $71 \pm 8$ years, FEV $152 \pm 16 \%$ predicted). Table 1 shows their main anthropometric, clinical and functional characteristics. Table 2 shows that patients walked a mean \pm SD $6663 \pm 4675$ steps $\cdot$ day $^{-1}$ and were physically active $\left(>5000\right.$ steps $\cdot$ day $^{-1}$ ) a median 4 days per week. During follow-up, 67 (38\%) patients suffered at least one COPD hospitalisation. Additionally, 10 patients died during follow-up without having had any hospitalisation; they exhibited lower physical activity levels in all variables than survivors (online supplementary table E1).

The number of daily steps and physically active days at baseline was lower in patients who were hospitalised during follow-up than in those who were not, while no differences were observed with regard to time in activity or intensity of physical activity (table 2). All physical activity variables decreased according to increased severity of airflow limitation in a statistically significant linear pattern (online supplementary table E2). When patients were stratified above or below the median of the average intensity of physical activity $(\geqslant 2.7 \mathrm{METs})$, we observed that the relationship between variables describing the quantity of physical activity and risk of COPD hospitalisations was restricted to those with low average intensity of physical activity (online supplementary table E3). Consequently, physical activity intensity was included as an interaction term in all subsequent analyses.

The multivariate Cox model, adjusted both for potential confounders and the interaction term between intensity and daily steps, showed that for every additional 1000 daily steps at low average intensity, the risk of COPD hospitalisation was reduced by $\sim 20 \%$ (hazard ratio (HR) 0.79 , 95\% CI 0.67-0.93; p=0.005) (table 3). However, increasing the number of daily steps when average intensity of physical activity was high did not result in any reduction in risk of COPD hospitalisation (table 3). This is illustrated in figure 1. Similar results were observed for the other two variables describing the quantity of physical activity (number of physically active days and time in activity) (table 3). After stratification according to airflow limitation, in patients with severe to very severe airflow limitation (FEV $1<50 \%$ pred) high quantity of activity at high average intensity was associated with a higher risk of COPD hospitalisation (fig. 2 and online supplementary table E4). Stratification of patients by hospitalisation for COPD in the previous year (online supplementary table E5), additional analysis using volume of physical activity as the exposure 
TABLE 1 Sociodemographic, clinical and functional characteristics of participants

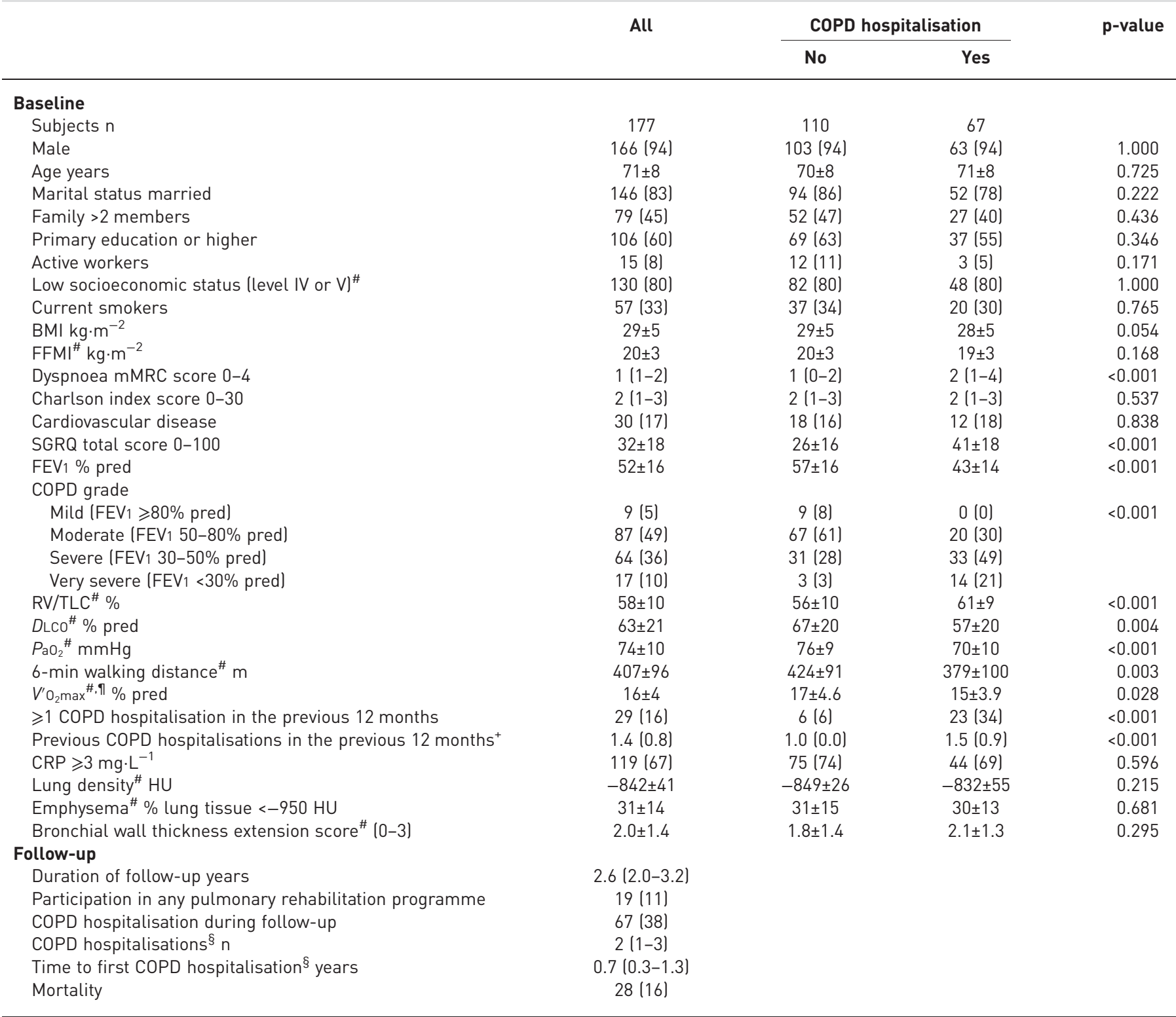

Data are presented as $\mathrm{n}, \mathrm{n}(\%)$, mean \pm SD or median (interquartile range), unless otherwise stated. COPD: chronic obstructive pulmonary disease; BMI: body mass index; FFMI: fat-free mass index; mMRC: modified Medical Research Council; SGRQ: St George's Respiratory Questionnaire; FEV1: forced expiratory volume in $1 \mathrm{~s}$; RV: residual volume; TLC: total lung capacity; DLCO: diffusing capacity of the lung for carbon monoxide; \% pred: \% predicted; $\mathrm{PaO}_{2}$ : arterial oxygen tension; $V^{\prime} \mathrm{O}_{2}$ max: maximal oxygen uptake; CRP: C-reactive protein. ${ }^{\#}$ : some variables have missing values: socioeconomic status ( $n=15), F F M I(n=13), R V / T L C$ ( $n=11), D L C O(n=17), P_{a O}(n=6), 6$-min walking distance ( $\left.n=10\right), V^{\prime} O_{2}$ max ( $\left.n=66\right)$, CRP ( $\left.n=11\right)$, lung density and emphysema $(n=112)$ and bronchial wall thickness $(n=96)$. Missing values were distributed at random and were mainly due to the hospital logistics and patient availability, as previously published [10]. " : during exercise testing using the reference values of NeDER et al. [13]; ${ }^{+}: n=29 ;{ }^{\S}: n=67$.

(online supplementary table E6), as well as the sensitivity analyses (online supplementary tables E7, E8, E9 and E10) all yielded similar results.

\section{Discussion}

The main finding of our study is that increased quantity of physical activity reduces the risk of future COPD hospitalisations when the average intensity of physical activity is low. The finding that high quantity of physical activity at high average intensity of physical activity may be unhelpful in COPD patients with severe to very severe airflow limitation was unexpected and will require replication. The sensitivity analyses showed that our results were very stable in relation to changes in the selection of subjects, variables and the use of different outcome measures. 
TABLE 2 Characteristics of patients' physical activity at baseline and according to hospitalisations for chronic obstructive pulmonary disease (COPD) during follow-up

\begin{tabular}{|c|c|c|c|c|}
\hline & \multirow[t]{2}{*}{ All patients } & \multicolumn{2}{|c|}{ COPD hospitalisation } & \multirow[t]{2}{*}{ p-value } \\
\hline & & No & Yes & \\
\hline Subjects & 177 & 110 & 67 & \\
\hline Physical activity steps-day ${ }^{-1}$ & $6663 \pm 4675$ & $7470 \pm 4939$ & $5339 \pm 3890$ & 0.003 \\
\hline Physically active days per week ${ }^{\#}$ & $4(1-7)$ & $5(2-7)$ & $2(0-7)$ & 0.007 \\
\hline Time in physical activity min $\cdot$ day $^{-1}$ & $176 \pm 123$ & $185 \pm 131$ & $160 \pm 108$ & 0.272 \\
\hline Average intensity of physical activity METs & $2.8 \pm 0.5$ & $2.8 \pm 0.5$ & $2.8 \pm 0.6$ & 0.258 \\
\hline
\end{tabular}

Data are presented as $n$, mean \pm SD or median (interquartile range), unless otherwise stated. METs: metabolic equivalent tasks. ${ }^{*}$ : defined as $\geqslant 5000$ steps day $^{-1}[12]$.

\section{Comparison with previous studies}

This is the first study that unravels the independent role of the quantity and the intensity of physical activity on the risk of COPD hospitalisations. Previous research consistently demonstrated that higher quantity of physical activity reduces the risk of future COPD exacerbation/hospitalisation [6]. However, some of these previous studies [14-16] measured the quantity of physical activity using variables of energy expenditure (MET-h per week or -kcal per day) that also included intensity in their calculation [17]. In view of the results of the study, this should be taken into account more carefully since it could reduce the beneficial effects of physical activity on COPD hospitalisation risk. It is worth noting that the only study that has used an objective tool (pedometer) to assess the quantity of physical activity found a risk reduction almost identical to that observed in the study (20\% for every additional 1000 steps) [18].

TABLE 3 Adjusted $^{\#}$ association between physical activity variables and hospitalisation for chronic obstructive pulmonary disease (COPD) (multivariate Cox proportional hazards regression]

\begin{tabular}{|c|c|c|c|}
\hline & $\operatorname{HR}(95 \% \mathrm{CI})$ & p-value & $R^{2}$ \\
\hline Subjects $n$ & 177 & & \\
\hline Steps per day (change for each 1000 steps per day) & $0.79(0.67-0.93)$ & 0.005 & 0.33 \\
\hline High average intensity of physical activity ( $\geqslant 2.7$ METs) & $2.71(1.27-5.81)$ & 0.010 & \\
\hline Interaction: stepsxintensity & $1.28(1.06-1.53)$ & 0.009 & \\
\hline$\geqslant 1$ COPD hospitalisation in the previous 12 months & $5.17(2.95-9.06)$ & $<0.001$ & \\
\hline FEV $1 \%$ pred & $0.96(0.95-0.98)$ & $<0.001$ & \\
\hline Physically active days per week (change for 1 day per week) & $0.79(0.67-0.93)$ & 0.005 & 0.32 \\
\hline High average intensity of physical activity ( $\geqslant 2.7$ METs) & $2.12(1.10-4.09)$ & 0.025 & \\
\hline Interaction: daysxintensity & $1.37(1.10-1.70)$ & 0.005 & \\
\hline$\geqslant 1$ COPD hospitalisation in the previous 12 months & $5.19(2.97-9.07)$ & $<0.001$ & \\
\hline FEV $1 \%$ pred & $0.96(0.94-0.98)$ & $<0.001$ & \\
\hline Time in physical activity (change for $1 \mathrm{~h}$ per day) & $0.79(0.63-0.99)$ & 0.039 & 0.31 \\
\hline High average intensity of physical activity ( $\geqslant 2.7$ METs) & $1.64(0.95-2.85)$ & 0.078 & \\
\hline Interaction: timexintensity & $1.36(1.01-1.82)$ & 0.041 & \\
\hline$\geqslant 1$ COPD hospitalisation in the previous 12 months & $5.51(3.16-9.62)$ & $<0.001$ & \\
\hline FEV $1 \%$ pred & $0.96(0.94-0.98)$ & $<0.001$ & \\
\hline
\end{tabular}

Data are presented as $n$, unless otherwise stated. An increase of 1000 steps per day at low average intensity of physical activity is related to reduced COPD hospitalisation risk (hazard ratio (HR) 0.79); high average intensity of physical activity without increasing the number of steps is related to increased COPD hospitalisation risk (HR 2.71); an increase of 1000 steps per day at high average intensity of physical activity is not related to COPD hospitalisation risk (HR 0.79×1.28=1.01). METs: metabolic equivalent tasks; FEV1: forced expiratory volume in $1 \mathrm{~s} .{ }^{\#}$ : other potential confounders (sex, age, education, marital status, family members, working status, socioeconomic status, inhaled bronchodilators or corticosteroids, smoking status, smoking duration and intensity, Charlson index, cardiovascular comorbidities, modified Medical Research Council dyspnoea score, health-related quality of life, forced vital capacity, residual volume/total lung capacity, diffusing capacity of the lung for carbon monoxide, arterial oxygen tension, body mass index, fat-free mass index, 6-min walking distance, maximal oxygen uptake, lung density and structure, C-reactive protein, tumour necrosis factor- $\alpha$, participation in a pulmonary rehabilitation programme and consumption of fruits, vegetables and cured meats) were not finally included in multivariate models because they did not relate to the outcome nor did they modify the coefficient estimate for the exposure $>10 \%$. 


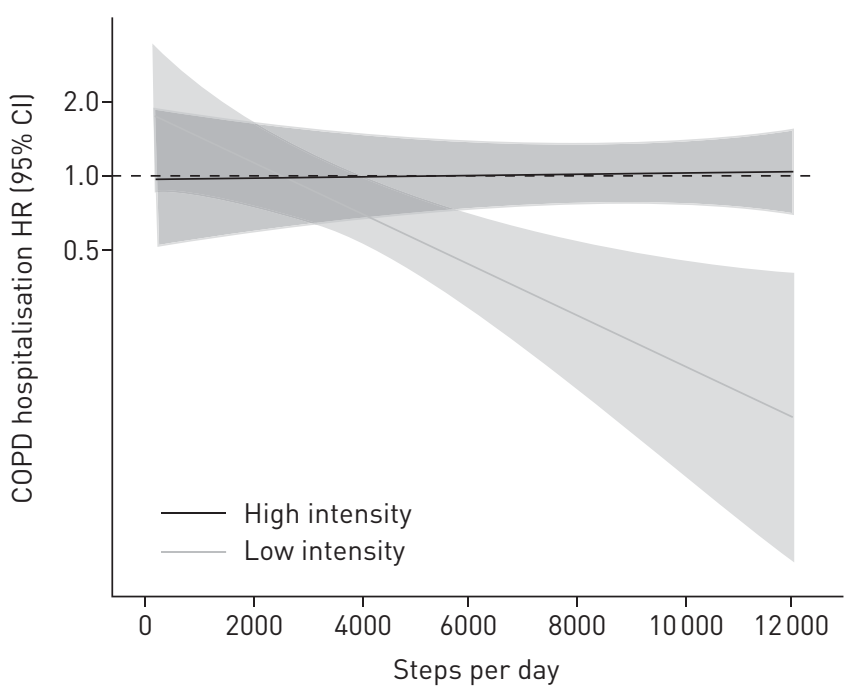

FIGURE 1 Dose-response relationship between steps per day and hazard ratio (HR) of hospitalisation for chronic obstructive pulmonary disease (COPD), according to intensity of physical activity (multivariate Cox proportional hazards regression). Hazard ratio was computed at each intensity value as the rate between hazard at each intensity value and average hazard of the sample. The model was adjusted for confounders as detailed in table 3.

The modifying effect of intensity of physical activity in the reported associations between its quantity and the risk of future hospitalisation is a novel finding in the COPD literature, although it has been previously reported in healthy elderly people, where health benefits were mainly related to low intensity of physical activity $[8,19,20]$. Specifically in the elderly, low-intensity physical activity has been shown to provide wellbeing benefits that were not found with moderate-to-vigorous physical activity [19]. It has been suggested that individuals engaging in more activities in the low intensity range (e.g. leisure-time walking) are more likely to have less stress, increased socialisation and greater quality of life [19]. Furthermore, low-intensity physical activity shows slight advantages over vigorous physical activity in reducing tumour necrosis factor- $\alpha$ and rising insulin-like growth factor binding protein-3 levels in aged people [20].

\section{Interpretation of findings}

The interpretation of the results for intensity of physical activity requires prior clarification. First, the intensity of physical activity is usually measured by METs, which express the ratio of energy expenditure
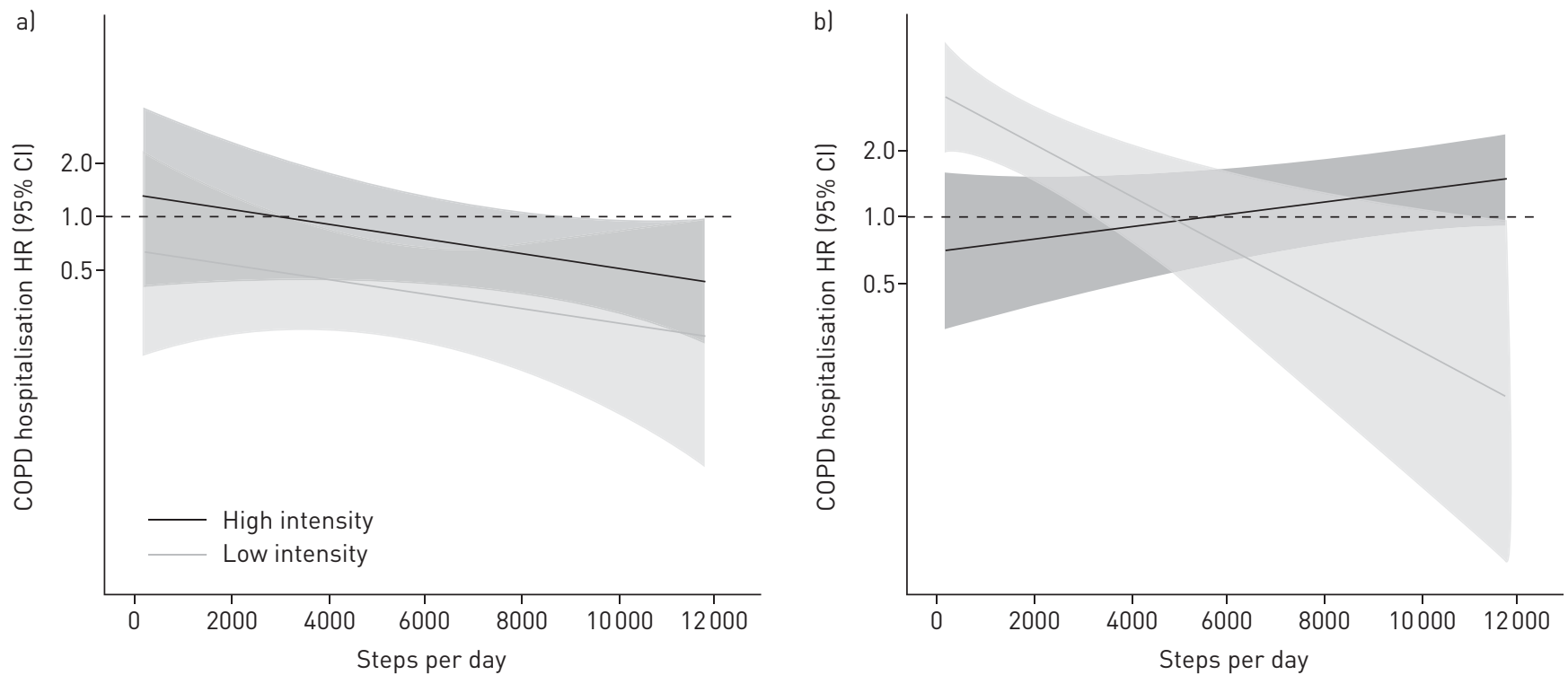

FIGURE 2 Dose-response relationship between steps per day and hazard ratio (HR) of hospitalisation for chronic obstructive pulmonary disease (COPD), according to intensity of physical activity and severity of airflow limitation: al mild to moderate (forced expiratory volume in $1 \mathrm{~s}$ (FEV 1 ) $\geqslant 50 \%$ pred); b) severe to very severe (FEV $1<50 \%$ pred). Hazard ratio was computed at each intensity value as the rate between hazard at each intensity value and the average hazard of the sample. The model was adjusted for confounders as detailed in table 3. 
during a specific physical activity in relation to the energy expenditure at rest. However, the relative metabolic demand (and, as a result, symptom perception) produced by a specific physical activity does not depend only on its MET value but also on the maximum intensity achievable by each subject. In fact, it is well known that for a given activity, COPD patients use a higher proportion of their metabolic and ventilatory capacities than their healthy peers [21]. Thus, the consideration about what is low- or highintensity physical activity differs depending on the population under study [17]. Second, it is also important to consider that the intensity was defined in our study as the average of METs during all periods spent in physical activity. This included basic activities of daily living, which represent the greater part of patients' time, and have an intensity of $\sim 1.5$ METs. Thus, to achieve a mean of 3 METs over 1 week, patients need to spend a large proportion of time at very high intensities most days of the week. Third, similarly to the framework that considers physical activity and exercise as different concepts [22], our average intensity of physical activity was conceptually different to an average intensity of exercise. Consequently, our results should not be interpreted as a contradiction of pulmonary rehabilitation programmes, which may include exercise of high intensity, because the impact of the latter on the daily average intensity of physical activity will be very small. In summary, our results support a beneficial effect of physical activity when the average of intensity is low, i.e. if only a small proportion of the daily time in physical activity is spent at high intensity levels. By contrast, when a large proportion of the time in physical activity is spent at higher intensities, physical activity appears no longer beneficial, a finding that will require replication.

Several mechanisms have been proposed as responsible for the beneficial effects of physical activity in COPD patients which could ultimately reduce the risk of exacerbations (and subsequent hospitalisations). First, moderate levels of physical activity have been shown to reduce the circulating levels of a number of inflammatory markers, both in experimental and observational studies, as well as in COPD populations [23-25]. Second, the regular practice of physical activity promotes more efficient oxygen delivery to respiratory muscles and improves the oxidative capacity of the muscles in COPD patients [26]. Regular physical activity has also been associated with higher levels of diffusing capacity of the lung for carbon monoxide, maximal expiratory pressure, 6-min walking distance and maximal oxygen uptake in COPD patients [25]. Studies on the effects of physical activity suggest that additional mechanisms may play a role in our observed increased risk of hospitalisation in the inactive patients, including 1) reduced nitric oxide production leading to deleterious vascular effects and loss of its bronchodilator effect [27]; 2) deregulation of gene expression of the $\beta_{2}$-adrenergic receptor, therefore possibly inducing a poorer response to therapy [28]; and 3) increased susceptibility to respiratory infections in COPD patients due to a reduction in the number and function of cells mediating cytotoxic activity [29].

Our novel findings of an unhelpful effect of higher quantity of high-intensity physical activity in severe to very severe COPD patients are supported by previous research in COPD patients, healthy subjects, elite athletes and animals. First, strenuous exercise is accompanied by an increase in circulating pro-inflammatory and inflammation-responsive cytokines [30], a response that is exaggerated in specific populations, such as overweight children [31]. In COPD patients, high-intensity exercise alters the nitroso-redox balance, which cannot be balanced by antioxidant mechanisms [26]. Studies in elite athletes have shown an association between high intensity of physical activity and increased airways inflammatory cells, as well as respiratory infections [32], which has been attributed to exercise-induced immune-suppressive effects of exercise, mostly affecting cell immunity. Finally, studies in animals suggest additional effects of high intensity of physical activity, such as the induction to apoptosis of inflammatory cells, mostly in elderly animals [33]. The complex role of physical activity in the immune system is still a matter of debate, more so in diseases with a high inflammatory component, such as COPD [29].

\section{Clinical implications of results}

Several of the observations of our study can have clinical implications for the recommendations about daily physical activity to COPD patients. This is an added value in the management of these patients, since no COPD-specific recommendations are available so far $[1,2]$. The linear dose-response relationship between quantity of low-intensity physical activity and the risk of COPD hospitalisations (fig. 2) suggests that there is no threshold, either low or high, to achieve clinical benefits. In addition to existing research [6], our results provide evidence to consider in the current COPD guidelines/strategies that COPD patients in general should be encouraged to perform an extra quantity of low-intensity physical activity during their daily life. Furthermore, the data suggest that research aiming to assess the effectiveness of physical activity interventions should consider several physical activity outcomes, including the proportion of individuals meeting certain physical activity goals [34], extra steps per day [35] and the changes, if any, in average intensity.

\section{Strengths and limitations}

Our study has several strengths and limitations. The extensive clinical, physiological and biological characterisation of participants within the framework of the PAC-COPD study [10] allowed the inclusion 
of potential confounders in the analysis, including history of previous COPD hospitalisations, which was not considered in previous studies $[15,16,36]$. Likewise, the use of an objective method to quantify physical activity (and the high compliance level) contrasts with previous studies that mainly used self-reported questionnaires [14, 15, 36, 37], reduces misclassification and allows the dissection of the role of different physical activity characteristics. In theory, our results could be explained by reverse causation, i.e. that hospitalisations reduce physical activity. Against this possibility is the fact that we recruited participants prospectively during their first COPD hospitalisation, that we followed them up for several years, that we restricted our current analysis to the next COPD hospitalisation and that we included the history of previous COPD hospitalisations as an adjusting variable. Another potential limitation of our study is the possibility of survival bias, namely that patients may die (and are therefore censored) before a COPD hospitalisation occurs. However, given that these patients performed low levels of physical activity (online supplementary table E1), the magnitude of the protective effect of physical activity on COPD hospitalisations is probably reduced. Likewise, the technological restrictions of the accelerometer to assess certain activities, such as cycling or swimming, could also be seen as a limitation. Similarly, the activity monitors can not yet be sufficiently accurate to identify small variations in the intensity of physical activities. However, it is now well recognised that accelerometers constitute the best tool to evaluate the quantity and intensity of physical activity during the activities of daily life; moreover, the specific accelerometer that we used is among the most valid [38]. It could also be argued that a single week of physical activity measurement may have resulted in some misclassification of patients' usual physical activity, because of season variation or low representativeness of that particular week. In either case, this would result in a reduction of the effect of physical activity. Our sample size was not large enough to assess the role of other physical activity characteristics such as frequency and duration of physical activity bouts, nor to assess the effects of physical activity on specific subgroups, such as patients with mild and very severe COPD. Finally, although the characteristics of our population, i.e. mainly retired, low socioeconomic status and having at least one previous COPD hospitalisation, may be characteristics that differ from other COPD cohorts, this probably does not affect the observed association of quantity and intensity of physical activity with COPD hospitalisations. In keeping with patterns of tobacco consumption in Europe, a majority of our patients were male; as there is evidence of sex-related differences in the effects of physical activity there is a need to replicate this study in females [39].

In conclusion, a greater quantity of low-intensity physical activity reduces the risk of COPD hospitalisation. The observation that high-intensity daily-life physical activity does not generate additional protective effects in the most severe COPD patients will require replication.

\section{Acknowledgements}

Members of the Phenotype Characterization and Course of COPD (PAC-COPD) Study Group are as follows. Josep M. Antó (Principal Investigator), Judith Garcia-Aymerich (project coordinator), Marta Benet, Jordi de Batlle, Ignasi Serra, David Donaire-Gonzalez and Stefano Guerra: Centre for Research in Environmental Epidemiology (CREAL), Barcelona, Spain; Joaquim Gea (centre coordinator), Eva Balcells, Àngel Gayete, Mauricio Orozco-Levi and Ivan Vollmer: Hospital del Mar-IMIM, Barcelona; Joan Albert Barberà (centre coordinator), Federico P. Gómez, Carles Paré, Josep Roca, Robert Rodriguez-Roisin, Àlvar Agustí, Xavier Freixa, Diego A. Rodriguez, Elena Gimeno-Santos and Karina Portillo: Hospital Clínic-Institut D’Investigacions Biomèdiques August Pi i Sunyer (IDIBAPS), Barcelona; Jaume Ferrer (centre coordinator), Jordi Andreu, Esther Pallissa and Esther Rodríguez: Hospital General Universitari Vall D’Hebron, Barcelona; Pere Casan (centre coordinator), Rosa Güell and Ana Giménez: Hospital de la Santa Creu i Sant Pau, Barcelona; Eduard Monsó (centre coordinator), Alicia Marín and Josep Morera: Hospital Universitari Germans Trias i Pujol, Badalona, Spain; Eva Farrero (centre coordinator) and Joan Escarrabill: Hospital Universitari de Bellvitge, Institut d'Investigació Biomèdica de Bellvitge (IDIBELL), L'Hospitalet de Llobregat, Spain; Antoni Ferrer (centre coordinator): Hospital de Sabadell, Corporació Parc Taulí, Institut Universitari Parc Taulí (Universitat Autònoma de Barcelona), Sabadell, Spain; Jaume Sauleda (centre coordinator) and Bernat Togores: Hospital Universitari Son Dureta, Palma de Mallorca, Spain; Juan Bautista Gáldiz (centre coordinator) and Lorena López: Hospital Universitario de Cruces, UPV, Barakaldo, Spain; José Belda: Instituto Nacional de Silicosis, Oviedo, Spain.

The authors acknowledge Milo Puhan (Johns Hopkins University, Baltimore, MD, USA) for his helpful comments on a previous version of the manuscript, Esther Gracia and David Martinez from the Centre for Research in Environmental Epidemiology (CREAL) for their help in extracting data from the accelerometers, and the Conjunto Mínimo Básico de Datos de Altas Hospitalarias (CMBDAH) from Catalonia, the Basque Country and the Balearic Islands for providing the information on hospitalisation data.

\section{References}

1 Vestbo J, Hurd SS, Agustí AG, et al. Global strategy for the diagnosis, management, and prevention of chronic obstructive pulmonary disease: GOLD executive summary. Am J Respir Crit Care Med 2013; 187: 347-365.

2 Celli BR, MacNee W, ATS/ERS Task Force. Standards for the diagnosis and treatment of patients with COPD: a summary of the ATS/ERS position paper. Eur Respir J 2004; 23: 932-946.

3 Sapey E, Stockley RA. COPD exacerbations. 2: aetiology. Thorax 2006; 61: 250-258.

4 Godtfredsen NS, Vestbo J, Osler M, et al. Risk of hospital admission for COPD following smoking cessation and reduction: a Danish population study. Thorax 2002; 57: 967-972. 
5 de Batlle J, Mendez M, Romieu I, et al. Cured meat consumption increases risk of readmission in COPD patients Eur Respir J 2012; 40: 555-560.

6 Gimeno-Santos E, Frei A, Steurer-Stey C, et al. Determinants and outcomes of physical activity in patients with COPD: a systematic review. Thorax 2014; 69: 731-739.

7 Garcia-Aymerich J, Serra Pons I, Mannino DM, et al. Lung function impairment, COPD hospitalisations and subsequent mortality. Thorax 2011; 66: 585-590.

8 Demakakos P, Hamer M, Stamatakis E, et al. Low-intensity physical activity is associated with reduced risk of incident type 2 diabetes in older adults: evidence from the English Longitudinal Study of Ageing. Diabetologia 2010; 53: 1877-1885.

9 Tanasescu M, Leitzmann MF, Rimm EB, et al. Exercise type and intensity in relation to coronary heart disease in men. JAMA 2002; 288: 1994-2000.

10 Garcia-Aymerich J, Gómez FP, Benet M, et al. Identification and prospective validation of clinically relevant chronic obstructive pulmonary disease (COPD) subtypes. Thorax 2011; 66: 430-437.

11 Donaire-Gonzalez D, Gimeno-Santos E, Balcells E, et al. Physical activity in COPD patients: patterns and bouts. Eur Respir J 2013; 42: 993-1002.

12 Tudor-Locke C, Craig CL, Thyfault JP, et al. A step-defined sedentary lifestyle index: <5000 steps/day. Appl Physiol Nutr Metab 2013; 38: 100-114.

13 Neder JA, Nery LE, Castelo A, et al. Prediction of metabolic and cardiopulmonary responses to maximum cycle ergometry: a randomised study. Eur Respir J 1999; 14: 1304-1313.

14 Garcia-Aymerich J, Farrero E, Félez MA, et al. Risk factors of readmission to hospital for a COPD exacerbation a prospective study. Thorax 2003; 58: 100-105.

15 Garcia-Aymerich J, Lange P, Benet M, et al. Regular physical activity reduces hospital admission and mortality in chronic obstructive pulmonary disease: a population based cohort study. Thorax 2006; 61: 772-778.

16 Garcia-Rio F, Rojo B, Casitas R, et al. Prognostic value of the objective measurement of daily physical activity in patients with COPD. Chest 2012; 142: 338-346.

17 Howley ET. Type of activity: resistance, aerobic and leisure versus occupational physical activity. Med Sci Sports Exerc 2001; 33: S364-S369.

18 Moy ML, Teylan M, Weston NA, et al. Daily step count predicts acute exacerbations in a US cohort with COPD. PloS One 2013; 8: e60400.

19 Buman MP, Hekler EB, Haskell WL, et al. Objective light-intensity physical activity associations with rated health in older adults. Am J Epidemiol 2010; 172: 1155-1165.

20 Onambélé-Pearson GL, Breen L, Stewart CE. Influence of exercise intensity in older persons with unchanged habitual nutritional intake: skeletal muscle and endocrine adaptations. Age 2010; 32: 139-153.

21 Vaes AW, Wouters EFM, Franssen FME, et al. Task-related oxygen uptake during domestic activities of daily life in patients with COPD and healthy elderly subjects. Chest 2011; 140: 970-979.

22 Caspersen CJ, Powell KE, Christenson GM. Physical activity, exercise, and physical fitness: definitions and distinctions for health-related research. Public Health Rep. 1985; 100: 126-131.

23 Handschin C, Spiegelman BM. The role of exercise and PGC1 $\alpha$ in inflammation and chronic disease. Nature 2008; 454: 463-469.

24 Di Raimondo D, Tuttolomondo A, Buttà C, et al. Metabolic and anti-inflammatory effects of a home-based programme of aerobic physical exercise. Int J Clin Pract 2013; 67: 1247-1253.

25 Garcia-Aymerich J, Serra I, Gómez FP, et al. Physical activity and clinical and functional status in COPD. Chest 2009; 136: 62-70.

26 Rabinovich RA, Ardite E, Troosters T, et al. Reduced muscle redox capacity after endurance training in patients with chronic obstructive pulmonary disease. Am J Respir Crit Care Med 2001; 164: 1114-1118.

27 Booth FW, Chakravarthy MV, Gordon SE, et al. Waging war on physical inactivity: using modern molecular ammunition against an ancient enemy. J Appl Physiol 2002; 93: 3-30.

28 Barr RG, Cooper DM, Speizer FE, et al. $\beta_{2}$-adrenoceptor polymorphism and body mass index are associated with adult-onset asthma in sedentary but not active women. Chest 2001; 120: 1474-1479.

29 Pedersen BK, Hoffman-Goetz L. Exercise and the immune system: regulation, integration, and adaptation. Physiol Rev 2000; 80: 1055-1081.

30 Pedersen BK, Ostrowski K, Rohde T, et al. The cytokine response to strenuous exercise. Can J Physiol Pharmacol 1998; 76: 505-511.

31 McMurray RG, Zaldivar F, Galassetti P, et al. Cellular immunity and inflammatory mediator responses to intense exercise in overweight children and adolescents. J Investig Med 2007; 55: 120-129.

32 Walsh NP, Gleeson M, Shephard RJ, et al. Position statement. Part one: immune function and exercise. Exerc Immunol Rev 2011; 17: 6-63.

33 Packer N, Hoffman-Goetz L. Apoptotic and inflammatory cytokine protein expression in intestinal lymphocytes after acute treadmill exercise in young and old mice. J Sports Med Phys Fitness 2012; 52: 202-211.

34 Spruit MA, Singh SJ, Garvey C, et al. An official American Thoracic Society/European Respiratory Society statement: key concepts and advances in pulmonary rehabilitation. Am J Respir Crit Care Med 2013; 188: e13-e64.

35 Mendoza L, Horta P, Espinoza J, et al. Pedometers to enhance physical activity in COPD: a randomised controlled trial. Eur Respir J 2015; 45: 347-354.

36 Benzo RP, Chang $\mathrm{CCH}$, Farrell $\mathrm{MH}$, et al. Physical activity, health status and risk of hospitalization in patients with severe chronic obstructive pulmonary disease. Respiration 2010; 80: 10-18.

37 Esteban C, Arostegui I, Aburto M, et al. Influence of changes in physical activity on frequency of hospitalization in chronic obstructive pulmonary disease. Respirology 2014; 19: 330-338.

38 Van Remoortel H, Raste Y, Louvaris Z, et al. Validity of six activity monitors in chronic obstructive pulmonary disease: a comparison with indirect calorimetry. PloS One 2012; 7: e39198.

39 Parker BA, Kalasky MJ, Proctor DN. Evidence for sex differences in cardiovascular aging and adaptive responses to physical activity. Eur J Appl Physiol 2010; 110: 235-246. 